\title{
Deficits in the miRNA-34a-regulated endogenous TREM2 phagocytosis sensor-receptor in Alzheimer's disease (AD); an update
}

\section{Surjyadipta Bhattacharjee, Yuhai Zhao and Walter J. Lukiw*}

Departments of Neurology, Neuroscience and Ophthalmology, Louisiana State University Neuroscience Center and Health Sciences Center, New Orleans, LA, USA *Correspondence: wlukiw@Isuhsc.edu

Edited and reviewed by:

Robert Marr, Rosalind Franklin University of Medicine and Science, USA

Keywords: Alzheimer's disease, miRNA-34a, TREM2, phagocytosis, innate-immune response, amyloidosis

One characteristic feature of Alzheimer's disease $(\mathrm{AD})$ neuropathology is the progressive generation, aggregation and deposition of the 42 amino acid amyloid beta (A $\beta 42)$ peptide, and other related amyloidogenic molecules, into dense clumps of insoluble, pro-inflammatory senile plaque cores in the extracellular space of the brain. It is not generally appreciated that the A $\beta 42$ peptide, derived via tandem betagamma secretase cleavage of the larger $\sim 770$ amino acid transmembrane betaamyloid precursor protein ( $\beta$ APP) is one of the "stickiest" peptides known, due in part to its high content of lipophillic and hydrophobic peptide domains $(21.4 \%$ valine-isoleucine; Mager, 1998; Watson et al., 2005). A $\beta 42$ peptide monomers have tremendously high potential for relatively rapid self-aggregation into $A \beta 42$ peptide dimers and higher order fibrillar structures via long-range, non-covalent hydrophobic forces that over time promote $\beta$ pleated sheet conformations (Mager, 1998; Watson et al., 2005; Boutajangout and Wisniewski, 2013; Chang et al., 2013). There is abundant evidence that under normal, homeostatic conditions, $\mathrm{A} \beta 42$ peptide monomers and perhaps other higher order $A \beta 42$ peptides are effectively "cleared" from the brain's extracellular medium by highly active and efficient innate-immune surveillance and phagocytic systems that limit excessive $\mathrm{A} \beta 42$ peptide dimerization, accumulation and further self-aggregation into pathological senile plaque lesions. Recently described, one of the key phagocytosis sensorreceptors responsible for $A \beta 42$ peptide clearance from the human central nervous system (CNS) is very likely the triggering receptor expressed in myeloid/microglial cells 2 (TREM2) enriched in myeloid cells and microglial cells of the CNS (Benitez et al., 2013; Forabosco et al., 2013; Gonzalez Murcia et al., 2013; Guerreiro et al., 2013; Neumann and Daly, 2013; Zhao et al., 2013; Jones et al., 2014; Figure 1). This short paper is an update on some very recent observations on TREM2 neurobiology, on newly discovered roles for miRNA-34a-mediated signaling in human degenerative disease, including miRNA-34a-mediated effects on TREM2 expression, and how dysfunctional TREM2 signaling may contribute to amyloidogenesis in AD and in related progressive, inflammatory neurodegenerative diseases of the human CNS.

TREM2 (TREM-2, Trem2a), a variably glycosylated 230 amino acid type 1 transmembrane sensor-receptor protein of the immunoglobulin gene superfamily is translated from a $\sim 1098$ nucleotide (nt) linear mRNA transcribed at chr6p21.1, is highly expressed in microglial cells of the human CNS (NCBI GenBank NG_011561; BC032362; http://www.genecards.org/cgi-bin/carddisp .pl?gene=TREM2; Zhao et al., 2013; Jones et al., 2014). As a recently recognized myeloid/microglial cell surface phagocytosis sensor-receptor, TREM2 appears to play a critical function in immune surveillance, the sensing of extracellular debris and phagocytosis throughout the CNS, including the recognition and ingestion of neurotoxic $A \beta 42$ peptides and other amyloidogenic extracellular debris (Benitez et al., 2013; Forabosco et al., 2013; Lattante et al., 2013; Neumann and Daly, 2013; Sieber et al., 2013; Hickman and Khoury, 2014; Jiang et al., 2014). TREM2 signaling is in part mediated through a tyrosine kinase-binding protein/DNAX activation adaptor protein of $12 \mathrm{kDa}$ (TYROBP/DAP12; Sieber et al., 2013; Zhao et al., 2013; Hickman and Khoury, 2014; Figure 1). Deficiencies in TREM2 abundance and/or function are associated with a defective innate-immune system, bone fragility, deficits in phagocytosis and amyloidogenesis, neurological alterations leading to presenile dementia in the autosomal recessive, inflammatory neurodegenerative disorder polycystic lipomembraneous osteodysplasia with sclerosing leukoencephalopathy (PLOSL; MIM 221770; Jarvi-Hakola-Nasu disease), and more recently with Parkinson's disease (PD), AD and amyotrophic lateral sclerosis (ALS; Nataf et al., 2005; Satoh et al., 2011; Benitez et al., 2013; Forabosco et al., 2013; Guerreiro et al., 2013; Neumann and Daly, 2013; Sieber et al., 2013; Zhao et al., 2013; Abduljaleel et al., 2014; Cady et al., 2014). Conversely, acute brain injury-induced increases in TREM2 expression in microglia suggests that TREM2 may contribute to neurotrophic roles after brain ischemia and this may impart a long term neurological benefit in functional recovery (Kawabori et al., 2013; Abduljaleel et al., 2014). Genome-wide association studies and meta-analysis (GWAS/MA) for AD have recently identified an $\mathrm{R} 47 \mathrm{H}$ (rs75932628) loss of function TREM2 variant as a significant risk factor for $\mathrm{AD}$, conveying an increase for $\mathrm{AD}$ with an odds ratio of 1.38.8 -fold ( $p=0.008$ ), an effect comparable to that of the e4 allele of the 299 amino acid APOE lipid transporter (Gonzalez 


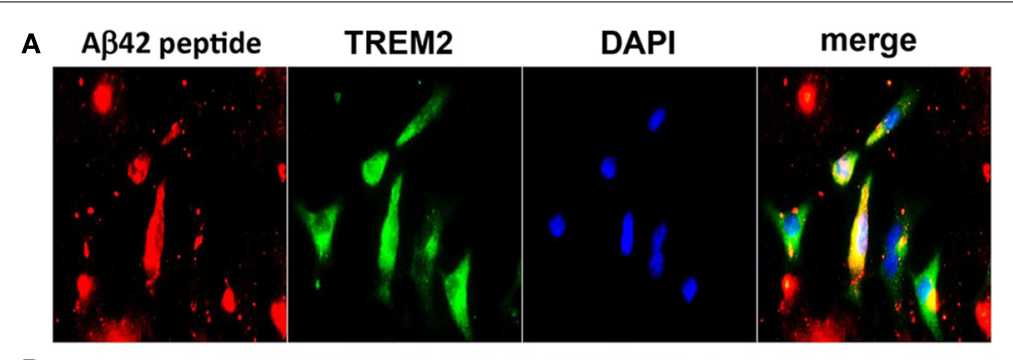

B

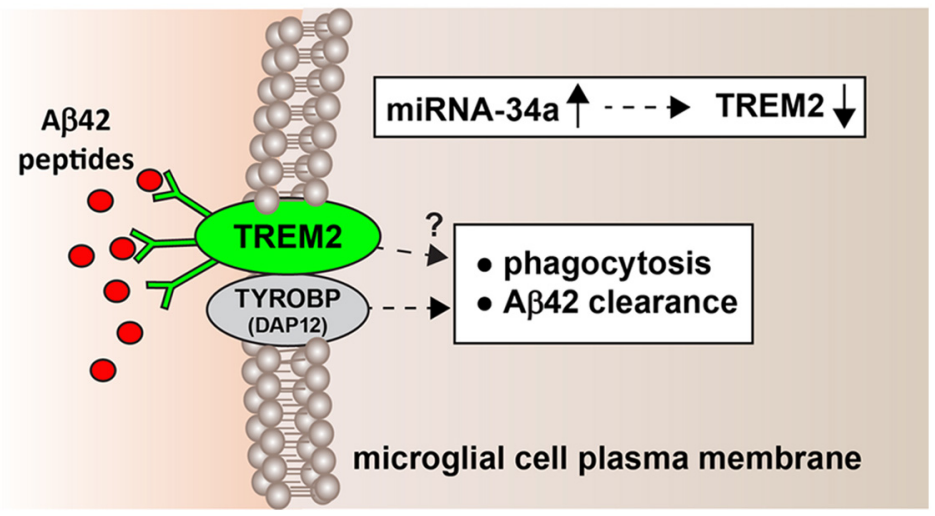

FIGURE 1 | (A) Murine microglial cell mediated phagocytosis of A $\beta 42$ peptides; 3 day old C8B4 murine microglia cells (ATCC CRL-2540; ATCC, Manassus VA) were treated with $5 \mu \mathrm{M}$ of $A \beta 42$ for $24 \mathrm{~h}$ before staining; A 342 peptides (American Peptide Company, Sunnyvale, CA, cat \# 62-0-80A) were prepared as described by Stine et al. (2003). Briefly, A $\beta 42$ peptides were initially solubilized in hexafluoroisopropanol (HFIP; Fluka Chemical, cat\# 52512; Sigma-Aldrich, St. Louis MO), aliquoted, and stored at $-20^{\circ} \mathrm{C}$ as an HFIP film. After vacuum evaporation of HFIP, aliquoted peptide was re-suspended with DMSO to $5 \mathrm{mM}$ and diluted to $5 \mu \mathrm{M}$ into the cell culture media; cells were subsequently stained using a murine amyloid beta MABN10 (red fluorescence $\lambda_{\max } \sim 650 \mathrm{~nm}$; anti-A $\beta$ antibody, clone W0-2; Millipore, Bellerica MA), a TREM-2 antibody (M-227): sc-48765 (green fluorescence; $\lambda_{\max } \sim 510 \mathrm{~nm}$; Santa Cruz, Santa Cruz CA) or DAPI nuclear stain; magnification $60 \times$; note self-aggregation of A 342 peptide after $24 \mathrm{~h}$ and $A \beta 42$ peptide affinity for TREM2 containing cells (leftmost panels) and internalization (rightmost panel; yellow merge; $\lambda_{\max } \sim 580 \mathrm{~nm}$ ); additional relevant methods have been described (Griciuc et al., 2013; Zhao and Lukiw, 2013); (B) highly schematicized depiction of the possible actions of an NF-kB-regulated, miRNA-34a-mediated TREM2 sensor-phagocytosis protein down-regulated in $A D$ brain; the triggering receptor for myeloid/microglial cells (TREM2) is a variably glycosylated transmembrane receptor known to be enriched in the microglial cell plasma membrane; signaling via the tyrosine kinase-binding protein (DNAX activation protein 12) [TYROBP (DAP12)] accessory receptor results in phagocytosis and ultimately, clearance of $A \beta 42$ peptides (red ovals) from the extracellular space (Satoh et al., 2011; Benitez et al., 2013; Forabosco et al., 2013; Hickman and Khoury, 2014); interestingly, TREM2 knockout/knockdown mice have attenuated immunological and inflammatory responses and/or increases in age-related neuroinflammatory markers and cognitive deficiency (Jiang et al., 2013; Sieber et al., 2013); TYROBP knockout mice exhibit immune system deficits and an impairment in microglial cell differentiation (Nataf et al., 2005; Sieber et al., 2013); it is not clear what, if any, contribution TREM2 makes directly to phagocytosis and A 342 clearance (question mark) without TYROBP (DAP12); while no deficits in TYROBP (DAP12) have been observed in sporadic AD brain, insufficient TREM2 may be in part responsible for the inability to adequately phagocytose $A \beta 42$ peptides, resulting in their buildup and self-aggregation in the extracellular space. Inset: miRNA-34a is found to be significantly increased in AD hippocampal CA1 and superior temporal lobe and in stressed microglial cells; miRNA-34a targeting of the TREM2 mRNA $3^{\prime}$-UTR appears to be in part responsible for this (see text); because miRNA-34a is encoded on an NF-kB-sensitive transcript, both anti-NF-kB and/or anti-miRNA strategies may be clinically useful in the restoration of homeostatic phagocytosis in the brain and CNS.
Murcia et al., 2013; Guerreiro et al., 2013; Neumann and Daly, 2013; Zhao et al., 2013). However, TREM2 R47H mutations appear to be relatively rare occurrences and may predispose only highly selective human populations to increased risk for age-related, pro-inflammatory neurodegenerative disorders such as $\mathrm{AD}$ (Gonzalez Murcia et al., 2013; Guerreiro et al., 2013; Hampel and Lista, 2013; Lattante et al., 2013; Bagyinszky et al., 2014). Indeed, AD cases are each highly complex, heterogeneous and multigenic neurodegenerative disorders and can be divided into those with a genetic or familial component ( $\sim 5 \%$ of all cases) or a sporadic or idiopathic form of $\mathrm{AD}$ with no known genetic cause $(95 \%$ of all cases; Blennow et al., 2006; Lukiw, 2013b; Bagyinszky et al., 2014). AD can be further classified as either early onset (under 65 years of age), or late-onset $\mathrm{AD}$ (LOAD; over 65 years of age; Bagyinszky et al., 2014; Rosenthal and Kamboh, 2014). Evidence for the loss of function $\mathrm{R} 47 \mathrm{H}$ mutation remains extremely rare in late onset $\mathrm{AD}$ in diverse human populations including relatively large, recent studies in Chinese
Han $(N=625)$ or Japanese $(N=4688)$ groups (Ma et al., 2014; Miyashita et al., 2014; Rosenthal and Kamboh, 2014). Indeed, much recent, independently derived data support the contention that the incidence of genetic mutations and epigenetic factors in $\mathrm{AD}$ varies widely amongst different human populations with different evolutionary backgrounds (Raj et al., 2010; Olson, 2012; Lukiw, 2013a,b). Interestingly, genetic-based loss-of-function mutations in TREM2 in LOAD may have the same end effect as a deficiency of a functional TREM2 in 
sporadic AD, with both pathways leading to compromised TREM2-mediated signaling and defective $A \beta 42$ sensing and removal by phagocytosis.

MicroRNAs (miRNAs) are small noncoding, single stranded RNAs (ssRNAs) that currently represent the smallest known carriers of highly selective genetic regulatory information in the human CNS (Lukiw, 2007; Hill et al., 2014a,b; Maffioletti et al., 2014). As highly mobile, autonomous genetic elements abundant in brain cell cytoplasm, the cerebrospinal fluid (CSF) and in the systemic circulation, miRNAs may be diagnostic for $\mathrm{AD}$ and other human CNS diseases (Alexandrov et al., 2012; Dorval et al., 2013). The primary mode of miRNA action is to recognize and bind to complementary ribonucleotide sequences in the 3-prime un-translated region ( $3^{\prime}$-UTR) of target messenger RNAs (mRNAs) and in doing so, down-regulate their expression (LagosQuintana et al., 2001; Lukiw et al., 2008; Guo et al., 2010). Several independent laboratories have recently reported the highly selective up-regulation of specific pathology-related miRNAs in $\mathrm{AD}$ brain including: (1) a significant up-regulation in the pro-inflammatory miRNA-34a (encoded at chr1p36.22) in $\mathrm{AD}$ and in $\mathrm{A} \beta 42$ peptide- and cytokine-stressed human primary brain cells (Cogswell et al., 2008; Lukiw, 2012; Zhao et al., 2013); (2) a significant miRNA-34a upregulation in amyloid overexpressing transgenic mouse models of $\mathrm{AD}$ (Wang et al., 2009; Zhao et al., 2013); (3) a productive and CNS-relevant miRNA34a-TREM2-3'-UTR interaction resulting in TREM2 deficits in cellular models of AD (Zhao and Lukiw, 2013; Zhao et al., 2013; Jones et al., 2014); and (4) an NFkB-mediated up-regulation of miRNA-34a coupled to a down-regulation of TREM2 in human neuronal-glial co-cultures (Alexandrov et al., 2013). The miRNA34a-mediated down-regulation of TREM2 appears to be due to an unusually strong miRNA-34a recognition feature within the 299 nt TREM2 mRNA 3'-UTR region (energy of association, $\mathrm{E}_{\mathrm{A}}<-16 \mathrm{kcal} / \mathrm{mol}$; http://www.genecards.org/cgi-bin/carddisp .pl?gene=TREM2; Zhao et al., 2013; Abduljaleel et al., 2014; Jiang et al., 2014; Jones et al., 2014). Interestingly, the stressand inflammation-induced transcription factor NF-kB, a driver for miRNA-34a expression, is also selectively up-regulated in $\mathrm{AD}$-affected brain regions, and both NF-kB inhibitors and stabilized antimiRNA-34a appear to be effective in restoring TREM2 back to homeostatic levels, at least in in vitro primary cell models of inflammatory neurodegeneration (Crampton and O'Keeffe, 2013; Lukiw, 2013a; Zhao and Lukiw, 2013; Zhao et al., 2013). Pathologically upregulated miRNA-34a-signaling has also been recently associated (1) with the repression of expression of several selected genes involved in cell survival and oxidative defense pathways such as $\mathrm{Bcl} 2$ and SIRT1 (Bhatnagar et al., 2014); (2) with spinal cord tissues in ALS (Cady et al., 2014); (3) with altered immunity associated with multiple sclerosis (Junker et al., 2009); (4) with progressive neurotrophic deficits including dysfunctional Bcl-2 signaling in transgenic murine models of AD (Wang et al., 2009); (5) with altered synaptogenesis (Agostini et al., 2011); (6) with deficient immune and phagocytotic responses in progressive inflammatory degeneration in cardiovascular disease (Boon et al., 2013); (7) with aging of the murine brain (Li et al., 2011); (8) with vasculature aging and cellular senescence (Boon et al., 2013; Agostini and Knight, 2014); (9) with blood mononuclear cells in sporadic AD patients (Schipper, 2007; Bhatnagar et al., 2014); (10) with lower mini-mental state examination (MMSE) scores when detected in the blood plasma of AD patients (Bhatnagar et al., 2014) and (11) with the progressive inflammatory neurodegeneration and epileptiform activities associated with epilepsy and the early stages of AD (Zhao et al., 2013; Henshall, 2014). The role of the CNS-enriched miRNA-34a and other pro-inflammatory miRNAs in epilepsy and AD is particularly interesting due to the overlapping neuropathology of these two neurological disorders with respect to the incidence of seizures and cognitive decline first apparent in the earliest stages of each disease (Vossel et al., 2013; Hill et al., 2014a,b).

Strengthening evidence continues to support the hypothesis that multiple genes, through multiple genetic processes, drive the initiation, propagation and course of sporadic AD.
Epigenetic mechanisms involving NF-кBmediated, miRNA-34a up-regulation and consequent down-regulation of TREM2 expression may drive the progressive extinction of the phagocytic response that in turn contributes to dysfunctional innate-immunity, amyloidogenesis and inflammatory neurodegeneration. Current data also suggest that the orchestrated interaction of at least two independent gene products on two different chromosomes-miRNA-34a at chr1p36.22 and TREM2 at chr6p21.1modulate TREM2 activities, the sensing of potentially hazardous waste molecules in the extracellular space, and the phagocytosis and clearance of this neurotoxic debris to maintain functional homeostasis in the CNS. Importantly, defective regulation of miRNA-34a and TREM2 signaling and other epigenetic effects on gene expression in sporadic $\mathrm{AD}$ would not be detectable via classical GWAS/MA or single nucleotide polymorphism (SNP) analysis of the genome (Hampel and Lista, 2013; Lukiw, 2013b). While dysfunction along the miRNA-34a-TREM2-TYOBP (DAP12) axis may be a particularly strong contributor to phagocytosis deficits and amyloidogenesis in $\mathrm{AD}$ it is important to note that other miRNA-mRNA pairings may also be involved in $A \beta 42$ clearance and altered innate-immune responses in this complex genetic regulatory network. AD-relevant stress-mediated increases in miRNA-34a in cultured brain cells, subsequent down-regulation in the expression of TREM2-3'-UTR reporter vectors, and rescue by anti-NF-kB or anti-miRNA34a pharmacological strategies indicates that TREM2 and accessory genetic signaling components that drive defective A 342 peptide sensing and phagocytosis can be effectively quenched, at least in in vitro studies (Lukiw, 2013b; Zhao et al., 2013; Jones et al., 2014). There is currently a great deal of pharmacological interest in the use of miRNA-34a mimics and their potential role in treating degenerative disease, including CNS disease and cancer, and miRNA-34a mimics have become the first half-life stabilized miRNAs to reach phase 1 clinical trials (Boon et al., 2013; Zhao et al., 2013; Agostini and Knight, 2014). Indeed anti-NF-kB, anti-miRNA-34a and/or analogous pharmacological molecular strategies may be 
useful in the future clinical management of $\mathrm{AD}$ and other multi-pathway neurological diseases with an amyloidogenic component, including novel combinatorial therapeutic approaches that have not yet been considered.

\section{ACKNOWLEDGMENTS}

Part of this study was presented at the "Non-Coding RNAs as Biomarkers and Diagnostics Symposium, Cambridge Healthtech Institute's 10th Annual Biomarkers \& Diagnostics World Congress," April 30-May 2, 2014 in Philadelphia PA, USA. Sincere thanks are extended to Drs. L. Carver, F. Culicchia, C. Eicken, E. Head and C. Hebel for short post-mortem interval (PMI) human brain biopsies, tissues or extracts, miRNA array analysis and preliminary data interpretation, and to D Guillot and AI Pogue for expert technical assistance. Thanks are also extended to the many physicians and neuropathologists of Canada and the USA who have provided high quality, short post-mortem interval human brain tissues for study. Additional human temporal lobe and other control and $\mathrm{AD}$ brain tissues were provided by the Memory Impairments and Neurological Disorders (MIND) Institute and the University of California, Irvine Alzheimer's Disease Research Center (UCI-ADRC; NIA P50 AG16573). The content of this manuscript is solely the responsibility of the authors and does not necessarily represent the official views of the NIH. Research on miRNA in the Lukiw laboratory involving the innate-immune response in $\mathrm{AD}$, amyloidogenesis and neuroinflammation was supported through a COBRE III Pilot Project, a Translational Research Initiative Grant from LSUHSC, the Louisiana Biotechnology Research Network (LBRN), an unrestricted departmental grant from Research to Prevent Blindness (RPB) and NIH Grants NEI EY006311, NIA AG18031 and NIA AG038834.

\section{REFERENCES}

Abduljaleel, Z., Al-Allaf, F. A., Khan, W., Athar, M., Shahzad, N., Taher, M. M., et al. (2014). Evidence of TREM2 variant associated with triple risk of Alzheimer's disease. PLoS ONE 9:e92648. doi: 10.1371/journal.pone.0092648

Agostini, M., and Knight, R. A. (2014). miR-34: from bench to bedside. Oncotarget 5, 872-881.

Agostini, M., Tucci, P., Killick, R., Candi, E., Sayan, B. S., Rivetti di Val Cervo, P., et al. (2011).
Neuronal differentiation by TAp73 is mediated by microRNA-34a regulation of synaptic protein targets. Proc. Natl. Acad. Sci. U.S.A. 108, 21093-21098. doi: 10.1073/pnas.1112061109

Alexandrov, P. N., Dua, P., Hill, J. M., Bhattacharjee, S., Zhao, Y., and Lukiw, W. J. (2012). microRNA (miRNA) speciation in Alzheimer's disease (AD) cerebrospinal fluid (CSF) and extracellular fluid (ECF). Int. J. Biochem. Mol. Biol. 3, 365-373.

Alexandrov, P. N., Zhao, Y., Jones, B. M., Bhattacharjee, S., and Lukiw, W. J. (2013). Expression of the phagocytosis-essential protein TREM2 is down-regulated by an aluminuminduced miRNA-34a in a murine microglial cell line. J. Inorg. Biochem. 128, 267-269. doi: 10.1016/j.jinorgbio.2013.05.010

Bagyinszky, E., Youn, Y. C., An, S. S., and Kim, S. (2014). The genetics of Alzheimer's disease. Clin. Interv. Aging 9, 535-551. doi: 10.2147/CIA.S51571

Benitez, B. A., Cruchaga, C., and United StatesSpain Parkinson's Disease Research Group. (2013). TREM2 and neurodegenerative disease. N. Engl. J. Med. 369, 1567-1568. doi: 10.1056/NEJMc1306509\#SA4

Bhatnagar, S., Chertkow, H., Schipper, H. M., Yuan, Z., Shetty, V., Jenkins, S., et al. (2014). Increased microRNA-34c abundance in Alzheimer's disease circulating blood plasma. Front. Mol. Neurosci. 7:2. doi: 10.3389/fnmol.2014.00002

Blennow, K., de Leon, M. J., and Zetterberg, H. (2006). Alzheimer's disease. Lancet 368, 387-403. doi: 10.1016/S0140-6736(06)69113-7

Boon, R. A., Lekushi, K., Lechner, S., Seeger, T., Fischer, A., Heydt, S., et al. (2013). MicroRNA-34a regulates cardiac ageing and function. Nature 495, 107-110. doi: 10.1038/nature11919

Boutajangout, A., and Wisniewski, T. (2013). The innate immune system in Alzheimer's disease. Int. J. Cell Biol. 2013:576383. doi: $10.1155 / 2013 / 576383$

Cady, J., Koval, E. D., Benitez, B. A., Zaidman, C., Jockel-Balsarotti, J., Allred, P., et al. (2014). TREM2 Variant p.R47H as a risk factor for sporadic amyotrophic lateral sclerosis. JAMA Neurol. 71, 449-453. doi: 10.1001/jamaneurol.2013.6237

Chang, C. C., Althaus, J. C., Carruthers, C. J., Sutton, M. A., Steel, D. G., and Gafni, A. (2013). Synergistic interactions between Alzheimer's A $\beta 40$ and $A \beta 42$ on the surface of primary neurons revealed by single molecule microscopy. PLoS ONE 8:e82139. doi: 10.1371/journal.pone. 0082139

Cogswell, J. P., Ward, J., Taylor, I. A., Waters, M., Shi, Y., Cannon, B., et al. (2008). Identification of miRNA changes in Alzheimer's disease brain and CSF yields putative biomarkers and insights into disease pathways. J. Alzheimers Dis. 14, 27-41. doi: 10.1016/j.jalz.2008.05.420

Crampton, S. J., and O'Keeffe, G. W. (2013). NF-кB: emerging roles in hippocampal development and function. Int. J. Biochem. Cell Biol. 45, 1821-1824. doi: 10.1016/j.biocel.2013.05.037

Dorval, V., Nelson, P. T., and Hébert, S. S. (2013). Circulating microRNAs in Alzheimer's disease: the search for novel biomarkers. Front. Mol. Neurosci. 6:24. doi: 10.3389/fnmol.2013.00024

Forabosco, P., Ramasamy, A., Trabzuni, D., Walker, R., Smith, C., Bras, J., et al. (2013). Insights into TREM2 biology by network analysis of human brain gene expression data. Neurobiol. Aging 34, 2699-2714. doi: 10.1016/j.neurobiolaging.2013.05.001

Gonzalez Murcia, J. D., Schmutz, C., Munger, C., Perkes, A., Gustin, A., Peterson, M., et al. (2013). Assessment of TREM2 rs75932628 association with Alzheimer's disease in a population-based sample: the Cache County Study. Neurobiol. Aging 34, 2889.e11-2889.e13. doi: 10.1016/j.neurobiolaging.2013.06.004

Griciuc, A., Serrano-Pozo, A., Parrado, A. R., Lesinski, A. N., Asselin, C. N., Mullin, K., et al. (2013). Alzheimer's disease risk gene CD33 inhibits microglial uptake of amyloid beta. Neuron 78, 631-643. doi: 10.1016/j.neuron.2013.04.014

Guerreiro, R., Wojtas, A., Bras, J., Carrasquillo, M., Rogaeva, E., Majounie, E., et al. (2013). TREM2 variants in Alzheimer's disease. N. Engl. J. Med. 368, 117-127. doi: 10.1056/NEJMoa1211851

Guo, H., Ingolia, N. T., Weissman, J. S., and Bartel, D. P. (2010). Mammalian microRNAs predominantly act to decrease target mRNA levels. Nature 466, 835-840. doi: 10.1038/nature09267

Hampel, H., and Lista, S. (2013). Have we learnt all we need to know from genetic studies - is genetics over in Alzheimer's disease? Alzheimers Res. Ther. 5:11. doi: 10.1186/alzrt165

Henshall, D. C. (2014). MicroRNA and epilepsy: profiling, functions and potential clinical applications. Curr. Opin. Neurol. 27, 199-205. doi: 10.1097/WCO.0000000000000079

Hickman, S. E., and El Khoury, J. (2014). TREM2 and the neuroimmunology of Alzheimer's disease. Biochem. Pharmacol. 88, 495-498. doi: 10.1016/j.bcp.2013.11.021

Hill, J. M., Bhattacharjee, S., Pogue, A. I., and Lukiw, W. J. (2014b). The gastrointestinal (GI) tract microbiome and potential link to Alzheimer's disease (AD). Front. Neurol. 5:43. doi: 10.3389/fneur.2014.00043

Hill, J. M., Zhao, Y., Bhattacharjee, S., and Lukiw, W. J. (2014a). miRNAs and viroids utilize common strategies in genetic signal transfer. Front. Mol. Neurosci. 7:10. doi: 10.3389/fnmol.2014.00010

Jiang, T., Yu, J. T., Zhu, X. C., and Tan, L. (2013). TREM2 in Alzheimer's disease. Mol. Neurobiol. 48, 180-185. doi: 10.1007/s12035-013-8424-8

Jiang, T., Yu, J. T., Zhu, X. C., Tan, M. S., Gu, L. Z., Zhang, Y. D., et al. (2014). Triggering receptor expressed on myeloid cells 2 knockdown exacerbates aging-related neuroinflammation and cognitive deficiency in senescence-accelerated mouse prone 8 mice. Neurobiol. Aging 35, 1243-1251. doi: 10.1016/j.neurobiolaging.2013.11.026

Jones, B. M., Bhattacharjee, S., Dua, P., Hill, J. M., Zhao, Y., and Lukiw, W. J. (2014). Regulating amyloidogenesis through the natural triggering receptor expressed in myeloid/microglial cells 2 (TREM2). Front. Cell. Neurosci. 8:94. doi: 10.3389/fncel.2014.00094

Junker, A., Krumbholz, M., Eisele, S., Mohan, H., Augstein, F., Bittner, R., et al. (2009). MicroRNA profiling of multiple sclerosis lesions identifies modulators of the regulatory protein CD47. Brain 132, 3342-3352. doi: 10.1093/brain/awp300

Kawabori, M., Hokari, M., Zheng, Z., Kim, J. Y., Calosing, C., Hsieh, C. L., et al. (2013). Triggering receptor expressed on myeloid cells- 2 correlates to hypothermic neuroprotection in ischemic stroke. 
Ther. Hypothermia Temp. Manag. 3, 189-198. doi: 10.1089/ther.2013.0020

Lagos-Quintana, M., Rauhut, R., Lendeckel, W., and Tuschl, T. (2001). Identification of novel genes coding for small expressed RNAs. Science 294, 853-858. doi: 10.1126/science.1064921

Lattante, S., Le Ber, I., Camuzat, A., Dayan, S., Godard, C., Van Bortel, I., et al. (2013). TREM2 mutations are rare in a French cohort of patients with frontotemporal dementia. Neurobiol. Aging 34, 2443.e1-2443.e2. doi: 10.1016/j.neurobiolaging.2013.04.030

Li, X., Khanna, A., Li, N., and Wang, E. (2011). Circulatory miR34a as an RNA-based, noninvasive biomarker for brain aging. Aging (Albany NY) 3 , 985-1002.

Lukiw, W. J. (2007). Micro-RNA speciation in fetal, adult and Alzheimer's disease hippocampus. Neuroreport 18, 297-300. doi: 10.1097/WNR.0b013e3280148e8b

Lukiw, W. J. (2012). NF-кB-regulated, proinflammatory miRNAs in Alzheimer's disease. Alzheimers Res. Ther. 4, 47. doi: 10.1186/alzrt150

Lukiw, W. J. (2013a). Antagonism of NF-kB-upregulated micro RNAs (miRNAs) in sporadic Alzheimer's disease (AD): anti-NF- $\mathrm{KB}$ vs. antimiRNA strategies. Front. Genet. 4:77. doi: 10.3389/fgene.2013.00077

Lukiw, W. J. (2013b) Variability in micro RNA (miRNA) abundance, speciation and complexity amongst different human populations and potential relevance to Alzheimer's disease (AD). Front. Cell. Neurosci. 7:133. doi: 10.3389/fncel.2013.00133

Lukiw, W. J., Zhao, Y., and Cui, J. G. (2008). An NF- $\kappa B$-sensitive micro RNA-146a-mediated inflammatory circuit in Alzheimer disease and in stressed human brain cells. J. Biol. Chem. 283, 31315-31322. doi: 10.1074/jbc.M8053 71200

Ma, J., Zhou, Y., Xu, J., Liu, X., Wang, Y., Deng, Y., et al. (2014). Association study of TREM2 polymorphism rs75932628 with lateonset Alzheimer's disease in Chinese Han population. Neurol. Res. 13:1743132814Y0000000376. doi: 10.1179/1743132814Y.0000000376

Maffioletti, E., Tardito, D., Gennarelli, M., and Bocchio-Chiavetto, L. (2014). Micro spies from the brain to the periphery: new clues from studies on microRNAs in neuropsychiatric disorders. Front. Cell. Neurosci. 8:75. doi: 10.3389/fncel.2014.00075
Mager, P. P. (1998). Molecular simulation of the primary and secondary structures of the Abeta (142)-peptide of Alzheimer's disease. Med. Res. Rev. $18,403-430$.

Miyashita, A., Wen, Y., Kitamura, N., Matsubara, E., Kawarabayashi, T., Shoji, M., et al. (2014). Lack of genetic association between TREM2 and lateonset Alzheimer's disease in a Japanese population. J. Alzheimers Dis. doi: 10.3233/JAD-140225. [Epub ahead of print].

Nataf, S., Anginot, A., Vuaillat, C., Malaval, L., Fodi, L. N., Chereul, E., et al. (2005). Brain and bone damage in KARAP/DAP12 loss-of-function mice correlate with alterations in microglia and osteoclast lineages. Am. J. Pathol. 166, 275-286. doi: 10.1016/S0002-9440(10)62251-1

Neumann, H., and Daly, M. J. (2013). Variant TREM2 as risk factor for Alzheimer's disease. N. Engl. J. Med. 368, 182-184. doi: 10.1056/ NEJMe1213157

Olson, M. V. (2012). Human genetic individuality. Аnnu. Rev. Genomics Hum. Genet. 13, 1-27. doi: 10.1146/annurev-genom-090711-163825

Raj, A., Rifkin, S. A., Andersen, E., and van Oudenaarden, A. (2010). Variability in gene expression underlies incomplete penetrance. Nature 463, 913-918. doi: 10.1038/nature08781

Rosenthal, S. L., and Kamboh, M. I. (2014). Lateonset Alzheimer's disease genes and the potentially implicated pathways. Curr. Genet. Med. Rep. 2, 85-101. doi: 10.1007/s40142-014-0034-x

Satoh, J., Shimamura, Y., and Tabunoki, H. (2011). Gene expression profile of THP-1 monocytes following knockdown of DAP12, a causative gene for Nasu-Hakola disease. Cell. Mol. Neurobiol. 32, 337-343. doi: 10.1007/s10571-011-9769-z

Schipper, H. M., Maes, O. C., Chertkow, H. M., and Wang, E. (2007). MicroRNA expression in Alzheimer blood mononuclear cells. Gene Regul. Syst. Bio. 1, 263-274.

Sieber, M. W., Jaenisch, N., Brehm, M., Guenther, M., Linnartz-Gerlach, B., and Neumann, H. (2013). Attenuated inflammatory response in triggering receptor expressed on myeloid cells 2 (TREM2) knock-out mice following stroke. PLoS ONE 8:e52982. doi: 10.1371/journal.pone.0052982

Stine, W. B., Jr., Dahlgren, K. N., Krafft, G. A., and $\mathrm{LaDu}, \mathrm{M}$. J. (2003). In vitro characterization of conditions for amyloid-beta peptide oligomerization and fibrillogenesis. J. Biol. Chem. 278, 11612-11622. doi: 10.1074/jbc.M210207200
Vossel, K. A., Beagle, A. J., Rabinovici, G. D., Shu, H., Lee, S. E., Naasan, G., et al. (2013). Seizures and epileptiform activity in the early stages of Alzheimer disease. JAMA Neurol. 70, 1158-1166. doi: 10.1001/jamaneurol.2013.136

Wang, X., Liu, P., Zhu, H., Xu, Y., Ma, C., Dai, X., et al. (2009). miRNA-34a, a microRNA up-regulated in a double transgenic mouse model of Alzheimer's disease, inhibits bcl2 translation. Brain Res. Bull. 80, 268-273. doi: 10.1016/j.brainresbull.2009.08.006

Watson, D., Castaño, E., Kokjohn, T. A., Kuo, Y. M., Lyubchenko, Y., Pinsky, D., et al. (2005). Physicochemical characteristics of soluble oligomeric $A \beta$ and their pathologic role in Alzheimer's disease. Neurol. Res. 27, 869-881. doi: 10.1179/016164105X49436

Zhao, Y., Bhattacharjee, S., Jones, B. M., Dua, P., Alexandrov, P. N., Hill, J. M., et al. (2013). Regulation of TREM2 expression by an NF-êB-sensitive miRNA-34a. Neuroreport 24, 318-323. doi: 10.1097/WNR.0b013e3283 $5 \mathrm{fb} 6 \mathrm{~b} 0$

Zhao, Y., and Lukiw, W. J. (2013). TREM2 signaling, miRNA-34a and the extinction of phagocytosis. Front. Cell. Neurosci. 7:131. doi: 10.3389/fncel.2013.00131

Conflict of Interest Statement: The authors declare that the research was conducted in the absence of any commercial or financial relationships that could be construed as a potential conflict of interest.

Received: 06 April 2014; accepted: 24 May 2014; published online: 17 June 2014.

Citation: Bhattacharjee S, Zhao Y and Lukiw WJ (2014)

Deficits in the miRNA-34a-regulated endogenous TREM2 phagocytosis sensor-receptor in Alzheimer's disease (AD); an update. Front. Aging Neurosci. 6:116. doi: 10.3389/fnagi.2014.00116

This article was submitted to the journal Frontiers in Aging Neuroscience.

Copyright (๑) 2014 Bhattacharjee, Zhao and Lukiw. This is an open-access article distributed under the terms of the Creative Commons Attribution License (CC BY). The use, distribution or reproduction in other forums is permitted, provided the original author(s) or licensor are credited and that the original publication in this journal is cited, in accordance with accepted academic practice. No use, distribution or reproduction is permitted which does not comply with these terms. 